\title{
Study and Implementation of Adaptive Meat in Core Network in PT Indosat Ooredoo
}

\author{
Yudhi Ardiyanto*1, Widyasmoro $^{1}$, Yessi Jusman ${ }^{1}$, Kharisma Fajar Sidik ${ }^{1}$ \\ ${ }^{1}$ Department of Electrical Engineering, Faculty of Engineering, Universitas Muhammadiyah \\ Yogyakarta \\ Bantul 55183 Daerah Istimewa Yogyakarta, Indonesia \\ *Corresponding author, e-mail: yudhi.ardiyanto@umy.ac.id
}

\begin{abstract}
Telecommunications technology is always developing every time from the beginning of the emergence of the first technology known as $1 G$ in 1980 until now its development is growing rapidly, in this year 2019 telecommunications technology is using $4 G$ technology that is sophisticated enough, to balance the sophistication, it must have efficient telecommunications procedures so that more optimal. One way to make the procedure more optimal, especially in paging, is by modifying the core network configuration located on the SGSN, from the initial use of the paging configuration to the adaptive paging configuration and prove whether it is more optimal by comparing the two configurations. The results of research that have been obtained from PT Indosat Ooredoo is that on SGSN Banjarmasin 4 (SGBJM4) an increase in the percentage of Paging Success Rate (PSR) of 0.96\%. In SGSN Banjarmasin 5 (SGBJM5), the percentage value increased 0.1\%. In SGSN Pontianak 1 (SGPTK1) an increase in the percentage value of $0.04 \%$. As for the RRC Paging Discard Ratio (PDR) decreased when using the adaptive paging configuration by $48.85 \%$.
\end{abstract}

Keywords: Telecommunications, Paging, Adaptive Paging, PSR, PDR

\section{Introduction}

Telecommunications technology is experiencing very rapid development from using $1 \mathrm{G}, 2 \mathrm{G}, 3 \mathrm{G}$, and now $4 \mathrm{G}$ technology. From the rapidly developing technological sophistication, it must have efficient telecommunications procedures so that it is more optimal. One way to make the procedure more optimal, especially in paging, is by modifying the core network configuration located on the SGSN, from the initial use of the paging configuration to the adaptive paging configuration and prove whether it is more optimal by comparing the two configurations. Therefore, to optimize this, a paging adjustment or referred to as adaptive paging with a low percentage of resources must be made but has the same or more success rate when using the paging configuration, so it will be more efficient.

Based on the topic of this research, there are a number of references of scientific research studies that have been carried out previously related to the objectives as a reference in this study. According to Guang Wan and Eric Lin. (1999). In his journal, entitled "Cost reduction in location management using semi-realtime movement information", he concluded that in this journal presents several paging and registration schemes based on semirealtime cellular terminal speed information for wireless communication networks. The main contribution of this journal is the proposed speed class concept, which provides more accurate runtime information for location tracking predictions. The concept of speed can be implemented based on existing registration schemes, such as movement-based registration or distance-based registration, with a minimum additional cost [1].

According to Kuan-PoLin and Hung-YuWe. (2009). In his journal entitled "Paging and Location Management in IEEE 802.16j Multihop Relay Network" has the conclusion that in this journal 
investigates the paging and location management scheme in the IEEE 802.16j multihop relay network. The paging scheme is compatible with standby mode operation in the IEEE $802.16 \mathrm{j}$ standard and integrates with the paging area design and timer-based location update mechanism scheme. The general random mobility model is suitable for investigating user mobility in multihop cellular relay systems, for example, IEEE 802.16j. Analytical mobility models are displayed to match the simulation results. This Junali applies this random road mobility model to analyze the proposed paging scheme. The proposed scheme performs well compared to the timency based scheme. In addition, optimization of the proposed paging area update has been demonstrated to effectively minimize signaling costs [2].

Aakanksha Sharma, Anurag Jain, and Anubhav Sharma. (2013). The journal entitled "An selective Paging Schemebased On Activity in Cellular Mobile Networks for Reporting Center" concluded that in this journal the main problem in cellular communication was finding the location of the cellular terminal at this time to provide services, known as Location Management (LM). LM involves tracking the current location, MT (Mobile Terminal's), which moves freely across different cells to provide services for them. This journal has applied predictive-based selective paging to the reporting center scheme in cellular networks, which reduces the cost of paging without affecting the cost of location renewal [3].

From a number of literature reviews that have been obtained from several previous scientific works discussing Cost Resource Paging, adaptive registration and paging schemes, optimization of paging by reducing the cost of paging without involving the latest location search. so that this thesis will develop adaptive paging on the core network at PT Indosat Ooredoo with the interview method regarding the final project to be made and take data regarding Paging Success Rate (PSR) and Radio Resource Control (RRC) based on the Resource used at PT Indosat Ooredoo, the discussion is to find out the level of success of paging or PSR, and to find out the value of the RRC after developing configuration on the SGSN from Paging to Adaptive paging configuration. The expectation is that although it reduces the value of the paging resource ratio, it does not involve tracking location when paging.

\section{Methods}

\section{Tools and Materials}

The tools and materials used for this final project are simple, using only software and hardware, including:

a. The software used is Microsoft Word 365, Microsoft Excel 365, Microsoft Visio, DbVisualizer Free 8.0.10, and Secure CRT.

b. The hardware used is a unit of HP Windows 10 Laptop with AMD Ryzen 52500 Processor specifications with Radeon VGA Vega Mobile Gfx 2.00 GHz RAM 4.00 GB, Flashdisk, and Printer.

\section{Flowchart of Research}

Based on Figure 1 Flowchart, it can be seen that the initial step taken is to study literature by studying references relating to the paper to be made so that it can identify existing problems and formulate problems, after which it can carry out data retrieval and subsequent processing data verification will be carried out.

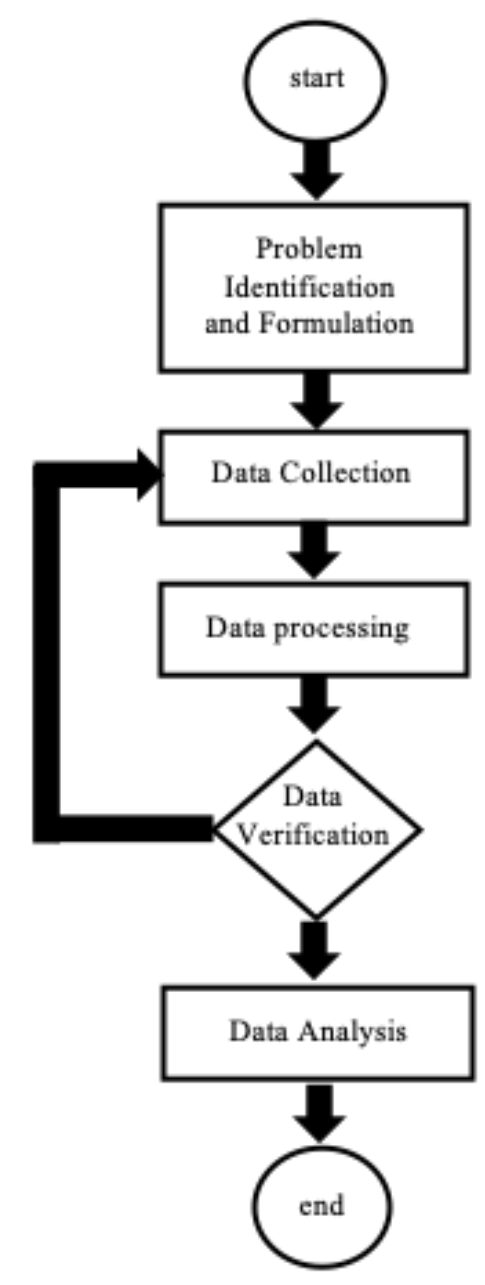

Fig. 1. Flowchart of research 
1. Problem Identification and Formulation Identification and formulation of the problem is the stage taken in the steps of this research that is identifying the main problems, the factors that cause problems that arise afterwards are interesting to identify, in identifying the problem by collecting data that has a relationship and influence against adaptive paging.

2. Data Collection

The data collection is divided into 2 types, namely primary data and secondary data.

a. Primary Data

Primary data is data obtained from the results of learning or direct observation of the materials needed in solving problems in this thesis. The method used in obtaining primary data is by interview or interview while at the location of data collection.

b. Secondary Data

Secondary data is data obtained by researchers from existing sources that have been collected by an agency or company.

3. Data Analysis

After obtaining the data to solve the formulation of the problem that has been made previously, then the next is to analyze the data that has been collected by making graphs of data when in the form of numbers or doing calculations and immediately conclude if the data collected has been in the form of graphs or the result of the total calculation. The data to be analyzed in the form of calculations or graphics for each parameter is affected when the configuration changes.

\section{Results}

\section{III.1. Flow Paging}

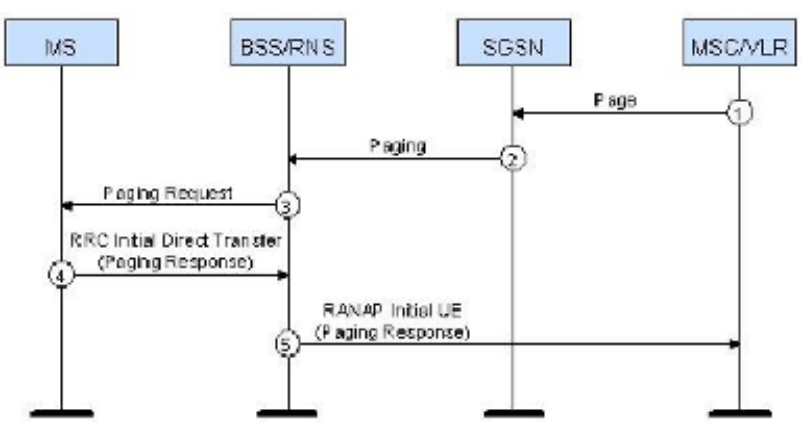

Fig. 2. Call setup paging

Based on Figure 2 the Call Setup paging that is triggered by MSC/VLR can be explained as follows:

4. SGSN receives paging messages (IMSI, VLR TMSI, Location Information) from MSC / VLR. If the TMSI VLR is removed, the IMSI is used instead of the TMSI as the paging address on the radio interface. If location information is not entered, SGSN must create MS pages in all cells served by VLR and SGSN, unless SGSN has reliable information about MS locations.

5. SGSN sends RANAP Paging (IMSI, TMSI, Area, Core Network (CN) Domain Indicator) messages to each Base Station System (BSS), BSS needs IMSI to calculate MS paging groups and to identify MS paging. TMSI is included if received from MSC.

6. BSS translates the PAN RANAP message that goes into a Radio Paging Request message and sends it to MS.

7. The CN Domain Indicator is set to 'CS' in the Initial Direct Transfer message.

8. When received at BSS, the Paging Response message is sent in the RANAP Initial UE message to MSC, which must stop the paging response timer later.

\section{III.2. Data Retrieval Results}

Retrieval of data that has been done is divided into 2 variables, namely the Paging Success Rate (PSR) data and the Paging Discard Ratio (PDR) data.

1. Paging Success Rate (PSR)

PSR is a ratio of the ratio between the number of paging successfully and the number of requests for paging. The formula used in calculating this ratio is:

$$
\mathrm{PSR}=(\text { Succes paging/Attach paging }) \times 100 \%
$$

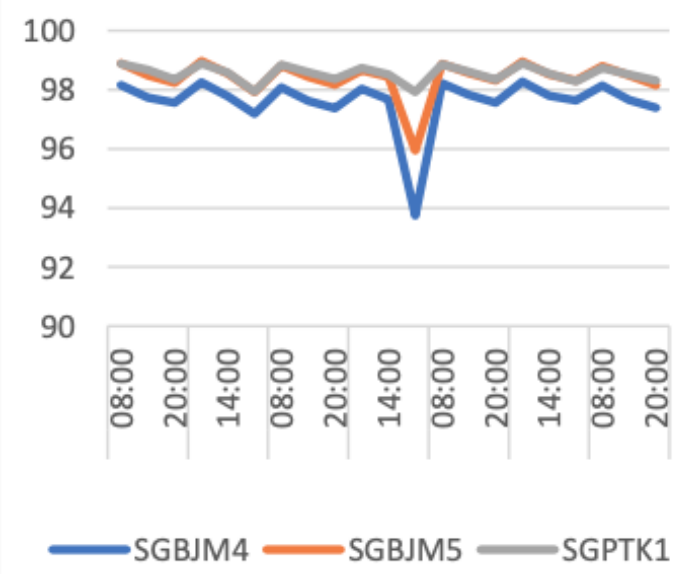

Fig. 3. Paging configuration PSR graph 


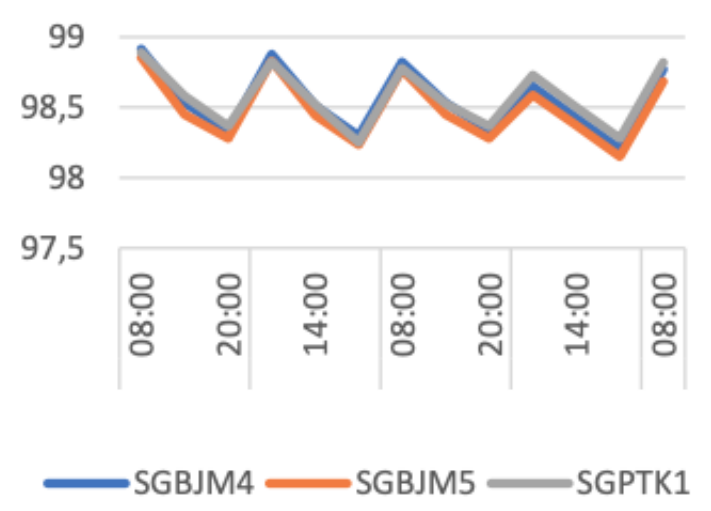

Fig. 4. Adaptive paging configuration PSR graph

Based on Figure 3 Graph PSR Paging Configuration and Figure 4 Adaptive Paging Configuration. In the two pictures it can be seen that there are 3 types of SGSN, namely GSBJM4 (SGSN Banjarmasin 4), SGBJM5 (SGSN Banjarmasin 5), and SGPTK1 (SGSN Pontianak 1). Based on these graphs, it can be concluded, among others:

a. On the SGBJM4 graph the Paging Success Rate (PSR) value increases when the adaptive paging configuration changes from the beginning using the paging configuration. When using the paging configuration the highest PSR value was $98.27 \%$ on November 6 , 2018, while the lowest PSR value was 93.74\% on November 4, 2018. After using the adaptive paging configuration the highest PSR value was $98.91 \%$ on the 8th November 2018 and the lowest PSR value was $98.20 \%$ on November 12, 2018. The average percentage value using the paging configuration was $97.61 \%$, while the average percentage value using the adaptive paging configuration was $98.58 \%$, thus the percentage increase the average value is $0.97 \%$.

b. On the SGBJM5 graph the Paging Success Rate (PSR) value increases when the adaptive paging configuration changes from the initial use of the paging configuration. When using the paging configuration the highest PSR value was $98.95 \%$ on November 6, 2018, while the lowest PSR value was $95.94 \%$ on November 4, 2018. After using the adaptive paging configuration the highest PSR value was 98.85\% on the 8th November 2018 and the lowest PSR value was $98.14 \%$ on November 11,2018 . The average percentage value using the paging configuration was $98.40 \%$, while the average percentage value using the adaptive paging configuration was $98.50 \%$, thus the percentage increase the average value is $0.1 \%$.

c. In the SGPTK1 chart the Paging Success Rate (PSR) value increases when the adaptive paging configuration was changed from the initial use of the paging configuration. When using the paging configuration the highest PSR value was $98.88 \%$ on November 2, 2018, while the lowest PSR value was $98.28 \%$ on November 6 2018. After using the adaptive paging configuration the highest PSR value was $98.89 \%$ on the 8 th November 2018 and the lowest PSR value was $98.25 \%$ on November 9, 2018. The average percentage value using the paging configuration was $98.54 \%$, while the average percentage value using the adaptive paging configuration was $98.58 \%$, thus the percentage value an average increase of about $0.04 \%$.

2. Paging Discard Ratio (PDR)

PDR is a ratio of the ratio between the number of discard RRC paging and the number of requests for RRC paging. The formula used in calculating this ratio is:

$$
\begin{aligned}
& \mathrm{PDR}=(10 \times(\text { Discard } \mathrm{RRC} \text { Paging } / \mathrm{RRC} \text { Paging } \\
&\text { Request })
\end{aligned}
$$

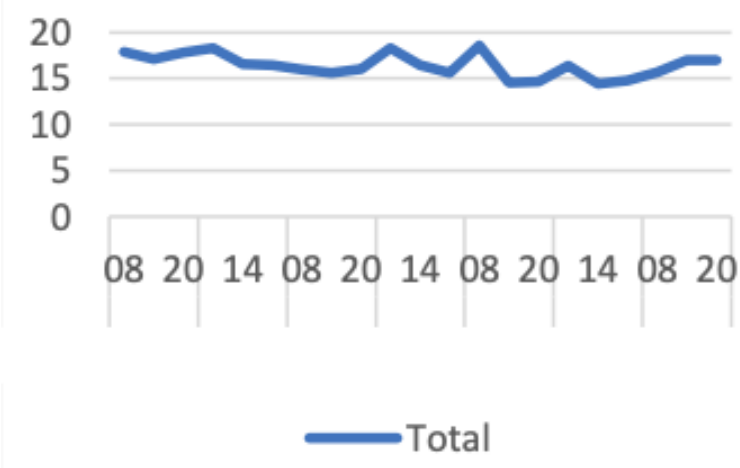

Fig. 5. Paging configuration PDR graph

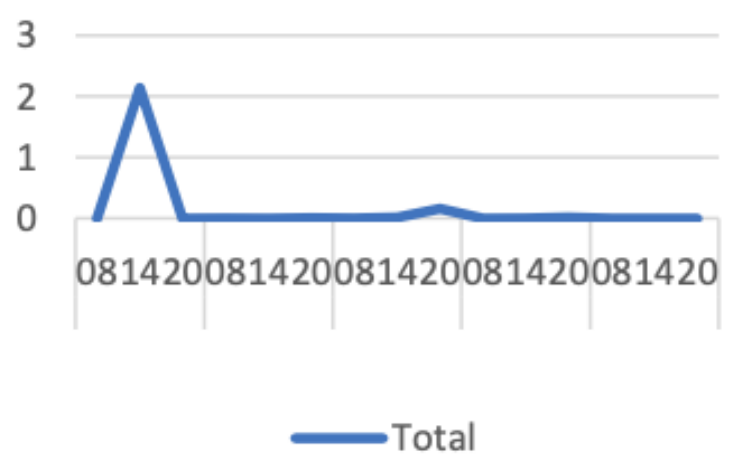

Fig. 6. Adaptive paging configuration PDR graph 
Based on Figure 5 and Figure 6, it can be seen that there is one line that illustrates the value of PDR. Based on these graphs, it can be concluded, that there was a change in the decrease in the value of the initial average percentage of $49.32 \%$ to $0.47 \%$, so that the percentage decreased as much as $48.85 \%$. The percentage decrease might be caused because when using adaptive paging, the resource used is small because of its narrow scope. Meanwhile, when using a paging resource configuration that is used a lot because of its broad scope.

\section{Conclusion}

Based on the results of the discussion of the analysis that has been carried out previously, then some conclusions can be drawn, including:

1. There is a change in the value of the percentage of Paging Success Rate (PSR) when a parameter change is made from the paging configuration to the adaptive paging configuration, ie the percentage value of the PSR has increased slightly or is fixed. In SGBJM4, the percentage of Paging Success Rate (PSR) increased by $0.97 \%$. In SGBJM5, the percentage value increased by $0.1 \%$. In SGPTK1, the percentage value increased by $0.04 \%$.

2. There is a change in the value of the percentage of the RRC Paging Discard Ratio (PDR) when a parameter change is made from the paging configuration to the adaptive paging configuration, ie a percentage decrease of $48.85 \%$.

\section{Acknowledgements}

This work was supported by Universitas Muhammadiyah Yogyakarta.

\section{References}

[1] G. Wan and E. Lin, "Cost reduction in location management using semi- realtime movement information," Wirel. Networks, vol. 5, no. 4, pp. 245-256, 1999.

[2] H. YuWe and K. PoLin, "Paging and Location Managementin IEEE 802.16j Multihop Relay Network," J. Comput. Syst. Networks, Commun. Taipei Hindawi Publ. Corp., vol. 2010, 2010.

[3] S. A. Jain and Anurag, "An Activity based Selective Paging Scheme for Reporting Centre in Cellular Mobile Networks," Int. J. Comput. Appl., vol. 66, no. 3, pp. 1-8, 2013.

\section{Authors' information}

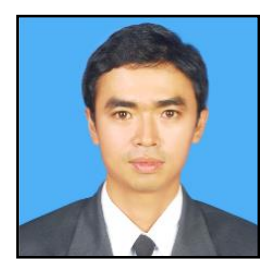

Yudhi Ardiyanto obtained his B. Eng in Electrical Engineering from Universitas Muhammadiyah Yogyakarta, Indonesia in 2008. His Master study was done at 2015 at the Electrical Engineering, Universitas Gadjah Mada, Indonesia. He currently is a lecture in department of electrical engineering, Universitas Muhammadiyah Yogyakarta.

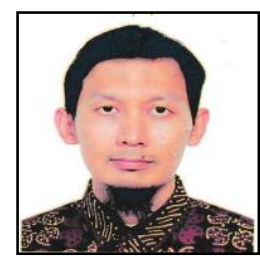

Widyasmoro obtained his B. Eng in Electrical Engineering from Universitas Jenderal Soedirman, Indonesia in 2007. His Master study was done at 2009 at the Electrical Engineering, Asia University, Taiwan. He currently is a lecture in department of electrical engineering, Universitas Muhammadiyah Yogyakarta.

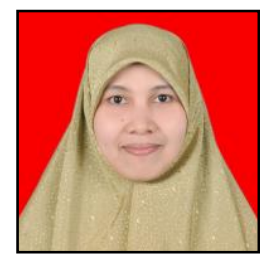

Yessi Jusman obtained her B. Eng in Electrical and Electronic Engineering from Andalas University, Indonesia in 2007. She worked as a Research Assistant started in July 2008 until November 2009 in Universiti Sains Malaysia. Her Master study was done at 2012 at the School of Electrical and Electronic Engineering, USM Engineering Campus in Nibong Tebal, Penang, Malaysia. She was finished her $\mathrm{PhD}$ degree at 2016 in University of Malaya with specializes in Image, Signal Processing, and algorithms.

Kharisma Fajar Sidik obtained his B. Eng in Electrical Engineering from Universitas Muhammadiyah Yogyakarta, Indonesia. 